NIKHEF-99-005

TTP99-08

\title{
Harmonic Polylogarithms
}

\author{
E. Remiddi ${ }^{a, b}$ and J. A. M. Vermaseren ${ }^{c}$ \\ a Institut für Theoretische TeilchenPhysik \\ University of Karlsruhe - D76128 Karlsruhe, Germany \\ Supported by the Alexander von Humboldt Stiftung \\ ${ }^{b}$ Dipartimento di Fisica, Università di Bologna, I-40126 Bologna, Italy \\ INFN, Sezione di Bologna, I-40126 Bologna, Italy \\ c NIKHEF, PO Box 41882, 1009DB Amsterdam, The Netherlands \\ e-mail: \\ remiddi@bo.infn.it \\ t68@nikhef.nl
}

\begin{abstract}
The harmonic polylogarithms (hpl's) are introduced. They are a generalization of Nielsen's polylogarithms, satisfying a product algebra (the product of two hpl's is in turn a combination of hpl's) and forming a set closed under the transformation of the arguments $x=1 / z$ and $x=(1-t) /(1+t)$. The coefficients of their expansions and their Mellin transforms are harmonic sums.
\end{abstract}

AMS(1991) subject classification: Primary 33B99, 33E99, Secondary 11Y60 Key words and phrases: Polylogarithms, Euler sums, harmonic series.

\section{Introduction.}

Euler's dilogarithm appeared very soon, even if with a different name, in the evaluation of radiative corrections in QED. The first occurrence is perhaps in the 1934 paper by G. Racah on the radiation by fast particles [1], whose function $F(x)$ is equal to $-\operatorname{Li}_{2}(-x)$ in Euler's notation. Two loop calculations [2] required the polylogarithms, Nielsen's generalization [3] of Euler's dilogarithm. 
More bibliographical indications as well as many relevant results are contained in the popular book by Lewin [4] (note the change in the titles of the two editions of the book).

While the polylogarithms are the natural analytical tool to use when dealing with the (relatively) simple integrals appearing in calculations with a few loops, it is known that they will not be sufficient when the number of loops will be larger than has been considered thus far or when several different scales are present. In a recent publication the set of polylogarithms has been extended into something called 'multidimensional polylogarithms' [5]. These functions seem to be very useful when more than one dimensionful parameter is involved. In principle they are a direct generalization of the definition of the power-series expansion of the polylogarithms to a multiparameter space.

Besides the dilogarithm, Euler studied also harmonic sums. A recent publication by one of us [6] investigated harmonic sums and their applicability, in particular to formulas in Mellin space. These harmonic sums seem to be the natural functions for the results of moment calculations of deep inelastic structure functions when only massless quarks are involved円. If indeed all these moments can be expressed in terms of harmonic sums, the class of functions that will represent the results in the regular $x$-space will be formed by the inverse Mellin transforms of these harmonic sums. In ref [6] it was indicated how one could obtain at least numerical representations of these functions by means of numerical integration.

In the current paper we study these functions in a more systematic way. We start with a recursive integral definition of a class of functions, which we will call the harmonic polylogarithms (hpl's), which are by construction a generalization of Nielsen's polylogarithms; it turns out, further, that an important subset of the hpl's is also a subset of the multidimensional polylogarithms of ref [5]. Then we will study a number of their properties, including expressions for products of harmonic polylogarithms with the same argument, the behaviour at $x=0,1$, the relevant expansions around those points, the algebra of the hpl's and the identities between hpl's of related arguments. Then we study special values and numerical evaluation. Finally we study the Mellin transforms of the harmonic polylogarithms and find that indeed they give the harmonic sums and that there is a one to one correspondence between them. As a consequence the investigation also leads to a rather simple algorithm for the inverse Mellin transform, even though in general the length of the resulting formulae requires a computer implementation for dealing with the great number of terms which are generated.

All algorithms that we present have been programmed in the language of FORM [0]. The resulting procedures can be obtained from the second author.

\footnotetext{
${ }^{1}$ This can be shown for all two loop calculations to any order in the expansion parameter $\epsilon$. For three loop calculations such results do not exist yet, but a recent result by Broadhurst and Kreimer shows that only at the 7-loop level the counter terms in the QCD beta function contains non-zeta like constants.
} 


\section{Definitions.}

The harmonic polylogarithms of weight $w$ and argument $x$ are identified by a set of $w$ indices, grouped into a $w$-dimensional vector $\vec{m}_{w}$ and are indicated by $\mathrm{H}\left(\vec{m}_{w} ; x\right)$.

More explicitly, for $w=1$ one defines

$$
\begin{aligned}
\mathrm{H}(0 ; x) & =\ln x \\
\mathrm{H}(1 ; x) & =\int_{0}^{x} \frac{d x^{\prime}}{1-x^{\prime}}=-\ln (1-x), \\
\mathrm{H}(-1 ; x) & =\int_{0}^{x} \frac{d x^{\prime}}{1+x^{\prime}}=\ln (1+x) .
\end{aligned}
$$

For their derivatives, one has

$$
\frac{d}{d x} \mathrm{H}(a ; x)=f(a ; x)
$$

where the index $a$ can take the 3 values $0,+1,-1$ and the 3 rational fractions $f(a ; x)$ are given by

$$
\begin{aligned}
f(0 ; x) & =\frac{1}{x}, \\
f(1 ; x) & =\frac{1}{1-x}, \\
f(-1 ; x) & =\frac{1}{1+x} .
\end{aligned}
$$

Note the (minor) asymmetry of Eq.(1), in contrast with the higher symmetry of Eq.(2).

For $w>1$, let us elaborate slightly the notation for the $w$-dimensional vectors $\vec{m}_{w}$. Quite in general, let us write

$$
\vec{m}_{w}=\left(a, \vec{m}_{w-1}\right),
$$

where $a=m_{w}$ is the leftmost index (taking of course one of the three values $0,1,-1$ ), and $\vec{m}_{w-1}$ stands for the vector of the remaining $(w-1)$ components. Further, $\overrightarrow{0}_{w}$ will be the vector whose $w$ components are all equal to the index 0 . The harmonic polylogarithms of weight $w$ are then defined as follows:

$$
\mathrm{H}\left(\overrightarrow{0}_{w} ; x\right)=\frac{1}{w !} \ln ^{w} x
$$

while, if $\vec{m}_{w} \neq \overrightarrow{0}_{w}$

$$
\mathrm{H}\left(\vec{m}_{w} ; x\right)=\int_{0}^{x} d x^{\prime} f\left(a ; x^{\prime}\right) \mathrm{H}\left(\vec{m}_{w-1} ; x^{\prime}\right) .
$$

Quite in general the derivatives can be written in the compact form

$$
\frac{d}{d x} \mathrm{H}\left(\vec{m}_{w} ; x\right)=f(a ; x) \mathrm{H}\left(\vec{m}_{w-1} ; x\right),
$$

where, again, $a=m_{w}$ is the leftmost component of $\overrightarrow{m_{w}}$. 
In analogy with Eq.(5), if $\overrightarrow{1}_{w},(\overrightarrow{-1})_{w}$ are the vectors whose components are all equal to 1 or -1 , we have by applying recursively the definitions

$$
\begin{aligned}
& \mathrm{H}\left(\overrightarrow{1}_{w} ; x\right)=\frac{1}{w !}(-\ln (1-x))^{w}, \\
& \mathrm{H}\left((-\overrightarrow{-1})_{w} ; x\right)=\frac{1}{w !} \ln ^{w}(1+x) .
\end{aligned}
$$

Let us now have a look at the first few values of the indices. For $w=2$ one has the 9 functions

$$
\begin{aligned}
\mathrm{H}(0,0 ; x) & =\frac{1}{2 !} \ln ^{2} x, \\
\mathrm{H}(0,1 ; x) & =\int_{0}^{x} \frac{d x^{\prime}}{x^{\prime}} \mathrm{H}\left(1 ; x^{\prime}\right)=-\int_{0}^{x} \frac{d x^{\prime}}{x^{\prime}} \ln \left(1-x^{\prime}\right), \\
\mathrm{H}(0,-1 ; x) & =\int_{0}^{x} \frac{d x^{\prime}}{x^{\prime}} \mathrm{H}\left(-1 ; x^{\prime}\right)=\int_{0}^{x} \frac{d x^{\prime}}{x^{\prime}} \ln \left(1+x^{\prime}\right), \\
\mathrm{H}(1,0 ; x) & =\int_{0}^{x} \frac{d x^{\prime}}{1-x^{\prime}} \mathrm{H}\left(0 ; x^{\prime}\right)=\int_{0}^{x} \frac{d x^{\prime}}{1-x^{\prime}} \ln x^{\prime}, \\
\mathrm{H}(1,1 ; x) & =\int_{0}^{x} \frac{d x^{\prime}}{1-x^{\prime}} \mathrm{H}\left(1 ; x^{\prime}\right)=-\int_{0}^{x} \frac{d x^{\prime}}{1-x^{\prime}} \ln \left(1-x^{\prime}\right), \\
\mathrm{H}(1,-1 ; x) & =\int_{0}^{x} \frac{d x^{\prime}}{1-x^{\prime}} \mathrm{H}\left(-1 ; x^{\prime}\right)=\int_{0}^{x} \frac{d x^{\prime}}{1-x^{\prime}} \ln \left(1+x^{\prime}\right), \\
\mathrm{H}(-1,0 ; x) & =\int_{0}^{x} \frac{d x^{\prime}}{1+x^{\prime}} \mathrm{H}\left(0 ; x^{\prime}\right)=\int_{0}^{x} \frac{d x^{\prime}}{1+x^{\prime}} \ln x^{\prime}, \\
\mathrm{H}(-1,1 ; x) & =\int_{0}^{x} \frac{d x^{\prime}}{1+x^{\prime}} \mathrm{H}\left(1 ; x^{\prime}\right)=-\int_{0}^{x} \frac{d x^{\prime}}{1+x^{\prime}} \ln \left(1-x^{\prime}\right), \\
\mathrm{H}(-1,-1 ; x) & =\int_{0}^{x} \frac{d x^{\prime}}{1+x^{\prime}} \mathrm{H}\left(-1 ; x^{\prime}\right)=\int_{0}^{x} \frac{d x}{1+x^{\prime}} \ln \left(1+x^{\prime}\right) .
\end{aligned}
$$

Those 9 functions can all be expressed in terms of logarithmic and dilogarithmic functions; indeed, if

$$
\operatorname{Li}_{2}(x)=-\int_{0}^{x} \frac{d x^{\prime}}{x^{\prime}} \ln \left(1-x^{\prime}\right)
$$

is the usual Euler's dilogarithm, one finds

$$
\begin{aligned}
\mathrm{H}(0,1 ; x) & =\mathrm{Li}_{2}(x) \\
\mathrm{H}(0,-1 ; x) & =-\mathrm{Li}_{2}(-x), \\
\mathrm{H}(1,0 ; x) & =-\ln x \ln (1-x)+\mathrm{Li}_{2}(x), \\
\mathrm{H}(1,1 ; x) & =\frac{1}{2 !} \ln ^{2}(1-x), \\
\mathrm{H}(1,-1 ; x) & =\mathrm{Li}_{2}\left(\frac{1-x}{2}\right)-\ln 2 \ln (1-x)-\mathrm{Li}_{2}\left(\frac{1}{2}\right), \\
\mathrm{H}(-1,0 ; x) & =\ln x \ln (1+x)+\mathrm{Li}_{2}(-x), \\
\mathrm{H}(-1,1 ; x) & =\mathrm{Li}_{2}\left(\frac{1+x}{2}\right)-\ln 2 \ln (1+x)-\mathrm{Li}_{2}\left(\frac{1}{2}\right), \\
\mathrm{H}(-1,-1 ; x) & =\frac{1}{2 !} \ln ^{2}(1+x) .
\end{aligned}
$$


Something similar happens for harmonic polylogarithms and Nielsen's polylogarithms of weight 3; that is no longer true however from weight $4 \mathrm{on}$. To make an example,

$$
\mathrm{H}(-1,0,0,1 ; x)=\int_{0}^{x} \frac{d x^{\prime}}{1+x^{\prime}} \operatorname{Li}_{3}\left(x^{\prime}\right)
$$

cannot be expressed in terms of Nielsen's polylogarithms of the same weight, even allowing for slightly more general arguments (i.e. when considering, besides $x$, also $-x,(1+x) / 2,(1-x) / 2$ etc.). In other words, the set of the $3^{w}$ harmonic polylogarithms of weight $w$ is in general a much wider set of functions than the set of the Nielsen's polylogarithms.

It follows from the definition that if $\vec{m}_{w} \neq \overrightarrow{0}_{w}$ the hpl's vanish at $x=0$ :

$$
\mathrm{H}\left(\vec{m}_{w} ; 0\right)=0, \quad \quad \vec{m}_{w} \neq \overrightarrow{0}_{w} .
$$

Likewise, if the leftmost index $m_{w}$ is not equal to $1,\left(m_{w} \neq 1\right), \mathrm{H}\left(\vec{m}_{w} ; 1\right)$ is finite; it is also finite when $\vec{m}_{w}=1$, but all the remaining indices $\vec{m}_{w-1}$ are zero, $\left(\vec{m}_{w-1}=\overrightarrow{0}_{w-1}\right)$. In the remaining cases, i.e. $\quad m_{w}=1$ and $\vec{m}_{w-1} \neq \overrightarrow{0}_{w-1}, \mathrm{H}\left(\vec{m}_{w} ; x\right)$ has a logarithmic behaviour at $x=1$ : more exactly, if the $p$ leftmost indices are all equal to $1, \mathrm{H}\left(\vec{m}_{w} ; x\right)$ behaves for $x \rightarrow 1$ as a combination of powers of $\ln (1-x)$ ranging from the maximum value $p$ down to 0 (the maximum power is decreased to $p-1$ if the remaining $w-p$ indices are all equal to zero; the study of the detailed logarithmic behaviours at $x=0,1$ will be carried out in Section 3 ).

In dealing with specific cases and except for the smallest values of $w$, specifying explicitly all the components of $\vec{m}$ becomes quite cumbersome, so that a more compact notation is welcome. In the case that we ignore the functions of which the last index is zero we can use the same compactified notation as in ref [6]. This is to say that, proceeding from right to left, all zeroes are simply eliminated by adding at the same time one to the absolute value of the previous index to the right, as in

$$
\mathrm{H}(0,0,1,0,-1 ; x)=\mathrm{H}_{3,-2}(x) .
$$

In terms of this notation and excluding, as already stated, the cases in which the rightmost index is zero, one can formulate the following:

theorem: If $m_{1} \neq 0$ one has

$$
\mathrm{H}_{m_{p}, \cdots, m_{1}}(-x)=(-1)^{p} \mathrm{H}_{-m_{p}, \cdots,-m_{1}}(x) .
$$

The proof goes by induction and follows rather trivially from the definition of $\mathrm{H}$. In the case that we use the notation in which the $m_{i}$ only have the value $0,1,-1$ the power of -1 is the number of indices that are not zero.

In general we will write the indices of the H-functions as subscripts when we use the notation of the r.h.s. of Eq.(14), while we will use the notation of the 1.h.s. when the indices are supposed to have the values $0,1,-1$ only. In that last notation, to see the relation with the polylogarithms of Nielsen $S_{n, p}(x)$, defined in [3], let us indicate with $\overrightarrow{0}_{n}, \overrightarrow{1}_{p}$ as usual, two $n$-dimensional and $p$-dimensional vectors whose components are all equal to 0 and 1 respectively; one then has

$$
S_{n, p}(x)=\mathrm{H}\left(\overrightarrow{0}_{n}, \overrightarrow{1}_{p} ; x\right) \text {. }
$$


As an obvious extension of the terminology, the product of two $\mathrm{H}$-functions of weight $w_{1}$ and $w_{2}$ will be said to have total weight $w=w_{1}+w_{2}$. In the following we will often encounter homogeneous "identities of weight $w$ ", i.e. relations (or identities) involving the sum of several terms, where each term is equal to the product of an integer or rational fraction times a $\mathrm{H}$-function of weight $w$ or a product of several H-functions separately of lower weight but with total weight $w$.

While the H-functions of weight $w$ are linear independent, the same is not true for the wider set of all the homogeneous expressions of weight $w$. The redundance can be used for establishing a number of (homogeneous) identities expressing a $\mathrm{H}$-function of some argument and weight $w$ as a homogeneous expression of the same weight involving $\mathrm{H}$-functions of the same or of related arguments (including constant arguments, such as for instance +1 or -1 ). The identities can be useful, typically, for exhibiting explicitly the behaviour at particular points (such as the logarithmic behaviour at 0 or \pm 1 ) or for obtaining relations between $\mathrm{H}$-functions of special arguments. Quite in general, while establishing such identities can be more or less wearisome, there is almost always a straightforward "standard method" for checking a given identity: one first verifies that the identity holds for a particularly convenient choice of the variable (or variables) and then differentiate it with respect to one of the arguments. In so doing one obtains another relation, however of lower weight, according to Eq.(7); the procedure can be iterated until a relation of weight 1 is eventually obtained, whose check is trivial (because the H-functions of weight 1 are just logarithms).

Likewise, also the mathematical constants corresponding to the particular values of the $\mathrm{H}$ function of weight $w$ (such as the values at $x=1$ when finite) can be given the same weight $w$. Those values, at $x= \pm 1$ or other simple arguments, are of particular interest by themselves, as it turns out that they can be expressed in terms of a very small number of mathematical constants, such as Riemann $\zeta$-functions, $\ln 2$ etc. We will see that they are connected to the sums to infinity of ref [6] which have been systematically evaluated and tabulated] by one of us (J.V.) to weight $=9$ and can be evaluated basically to any weight, given enough computer resources?. In similar ways these sums have been evaluated under the name of Euler/Zagier sums by the authors of ref [9]. Hence, whenever H-functions at $x=1$ will appear in this paper they can be regarded as known from ref [6] or ref [9], provided their weight is not too large. It will also be shown that one may alternatively consider them as unknown constants, to be expressed in terms of that much smaller number of mathematical constants by systematically exploiting the many identities among H's of various arguments established in the rest of this paper.

\footnotetext{
${ }^{2}$ in ref [6] this was done only to weight $=7$

${ }^{3}$ An alternative method to obtain the finite constants consists of their numerical evaluation to high precision and then fitting them to a presumed basis. Using this method Broadhurst 11 has evaluated all finite objects at weight $=9$ and some objects at the weights 10 and 11
} 


\section{Identities between functions of the same argument.}

Let us start by the integration by parts (ibp) identities. From the very definition,

$$
\begin{aligned}
\mathrm{H}\left(m_{1} \cdots m_{q} ; x\right) & =\int_{0}^{x} d x^{\prime} f\left(m_{1} ; x^{\prime}\right) \mathrm{H}\left(m_{2} \cdots m_{q} ; x^{\prime}\right) \\
& =\mathrm{H}\left(m_{1} ; x\right) \mathrm{H}\left(m_{2} \cdots m_{q} ; x\right)-\int_{0}^{x} d x^{\prime} \mathrm{H}\left(m_{1} ; x^{\prime}\right) f\left(m_{2} ; x^{\prime}\right) \mathrm{H}\left(m_{3} \cdots m_{q} ; x^{\prime}\right) \\
& =\mathrm{H}\left(m_{1} ; x\right) \mathrm{H}\left(m_{2} \cdots m_{q} ; x\right)-\mathrm{H}\left(m_{2} m_{1} ; x\right) \mathrm{H}\left(m_{3} \cdots m_{q} ; x\right) \\
& +\mathrm{H}\left(m_{3} m_{2} m_{1} ; x\right) \mathrm{H}\left(m_{4} \cdots m_{q} ; x\right)-\cdots-(-1)^{p} \mathrm{H}\left(m_{q} \cdots m_{1} ; x\right) .
\end{aligned}
$$

The above identity can be immediately verified, independently of its derivation, by the 'standard methods': it holds at $x=0$; when differentiating with respect to $x$, one obtains a number of terms which are immediately seen to cancel out pairwise; therefore, the relation is true. This relation shows that in the case that $\vec{m}_{q}$ is symmetric and $q$ is even the $\mathrm{H}$-function reduces to products of lower weight functions. In general the relation can be used when it is important to reduce the number of $\mathrm{H}$-functions with the highest weight as much as possible.

Another important set of identities expresses the product of any two $\mathrm{H}$-functions of weight $w_{1}$ and $w_{2}$ as a linear combination of $\mathrm{H}$-functions of weight $w=w_{1}+w_{2}$. Let us start from the case $w_{1}=1 ;$ the identity reads

$$
\begin{aligned}
\mathrm{H}(a ; x) \mathrm{H}\left(m_{p}, \cdots, m_{1} ; x\right) & =\mathrm{H}\left(a, m_{p} \cdots, m_{1} ; x\right) \\
& +\mathrm{H}\left(m_{p}, a, m_{p-1} \cdots, m_{1} ; x\right) \\
& +\mathrm{H}\left(m_{p}, m_{p-1}, a, m_{p-2} \cdots m_{1} ; x\right) \\
& +\cdots \\
& +\mathrm{H}\left(m_{p}, \cdots, m_{1}, a ; x\right) .
\end{aligned}
$$

It can be established by induction in $p$. For $p=1$ it is almost trivial, corresponding to Eq.(17) for $q=2$. Assume then that it holds for $p-1$; take the identity for $p-1$, multiply by $f\left(m_{p} ; x\right)$ and integrate over $x$. In the r.h.s. we can do the integral and obtain all necessary terms except for the one starting with $a$. The 1.h.s. can be integrated by parts to give the proper l.h.s. term plus another term that can be integrated and gives indeed the missing term. This completes the proof.

Again, once established the identity can also be verified by the 'standard method': it holds at $x=0$; the $x$-derivative consists of two groups of terms, a first group with the coefficient $f(a ; x)$ contains just two terms which cancel out immediately, plus a second group proportional to $f\left(m_{p} ; x\right)$, which is nothing but the same relation at level $p-1$, so that the procedure can be repeated $p$ times until everything cancels out.

There is only one complication with Eq.(18). This concerns points in which one of the objects involved is divergent. Hence one cannot apply this equation for $x=1$ in the case that either $a=1$ or $m_{p}=1$. This is explained better in the section on the algebraic properties.

Eq.(18) can be generalized to the product of two $\mathrm{H}$-functions $\mathrm{H}(\vec{p} ; x) \mathrm{H}(\vec{q} ; x)$; if $p, q$ are the dimensions of $\vec{p}, \vec{q}$ (or, which is the same, the weights of the two H-functions), the product is equal 
to the sum of $(p+q) ! / p ! q$ ! terms, each term being an H-function of weight $(p+q)$ with coefficient +1 , obtained by choosing $p$ indices in all possible ways (hence the binomial coefficients) and filling them from left to right with the components of $\vec{p}$ without changing their order, while the remaining $q$ places contain the components of $\vec{q}$, again without altering their order. This can be expressed with the formula

$$
\mathrm{H}(\vec{p} ; x) \mathrm{H}(\vec{q} ; x)=\sum_{\vec{r}=\vec{p} \uplus \vec{q}} \mathrm{H}(\vec{r} ; x)
$$

in which $\vec{p} \uplus \vec{q}$ represents all mergers of $\vec{p}$ and $\vec{q}$ in which the relative orders of the elements of $\vec{p}$ and $\vec{q}$ are preserved.

As an example, for $p=2, \vec{p}=(a, b)$ and $q=3, \vec{q}=(r, s, t)$ one has

$$
\begin{aligned}
\mathrm{H}(a, b ; x) \mathrm{H}(r, s, t ; x) & =\mathrm{H}(a, b, r, s, t ; x)+\mathrm{H}(a, r, b, s, t ; x) \\
& +\mathrm{H}(a, r, s, b, t ; x)+\mathrm{H}(a, r, s, t, b ; x) \\
& +\mathrm{H}(r, a, b, s, t ; x)+\mathrm{H}(r, a, s, b, t ; x) \\
& +\mathrm{H}(r, s, a, b, t ; x)+\mathrm{H}(r, a, s, t, b ; x) \\
& +\mathrm{H}(r, s, a, t, b ; x)+\mathrm{H}(r, s, t, a, b ; x),
\end{aligned}
$$

as can be easily checked, again, by the 'standard method'.

The product identities Eq.(19) can be used to single out the terms in $\ln (x)$ from H-functions whose indices have trailing (or rightmost) indices equal to zero (as we will see in the next section $\mathrm{H}$-functions with no trailing zeroes can be expanded in series of $x$ around $x=0$, while H-functions with trailing zeroes develop logarithmic singularities at that point). For $a=0$ in Eq.(18), recalling $\mathrm{H}(0 ; x)=\ln (x)$, Eq.(5) and Eq.(1) one obtains

$$
\begin{aligned}
\mathrm{H}\left(m_{1}, \cdots, m_{p}, 0 ; x\right)= & \ln (x) \mathrm{H}\left(m_{1}, \cdots, m_{p} ; x\right)-\mathrm{H}\left(0, m_{1}, \cdots, m_{p} ; x\right) \\
& -\mathrm{H}\left(m_{1}, 0, m_{2}, \cdots, m_{p} ; x\right)-\cdots-\mathrm{H}\left(m_{1}, \cdots, m_{p-1}, 0, m_{p} ; x\right) .
\end{aligned}
$$

In the case that $m_{p}$ is also zero we can move the last term to the left, divide by two and then use again Eq.(18) for all the other terms, thus obtaining an identity which extracts the logarithmic singularities due to 2 trailing zeroes. By suitably repeating the procedure as many times as needed, we can extract in general all the powers of $\ln (x)$ from the generic H-function. A couple of examples, if $a, b$ are any non-zero indices, are

$$
\begin{aligned}
\mathrm{H}(a, b, 0,0 ; x) & =\mathrm{H}(0,0 ; x) \mathrm{H}(a, b ; x) \\
& -\mathrm{H}(0 ; x)(\mathrm{H}(a, 0, b ; x)+\mathrm{H}(0, a, b ; x)) \\
& +\mathrm{H}(a, 0,0, b ; x)+\mathrm{H}(0, a, 0, b ; x)+\mathrm{H}(0,0, a, b ; x), \\
\mathrm{H}(a, b, 0,0,0 ; x) & =\mathrm{H}(0,0,0 ; x) \mathrm{H}(a, b ; x) \\
& -\mathrm{H}(0,0 ; x)(\mathrm{H}(a, 0, b ; x)+\mathrm{H}(0, a, b ; x))
\end{aligned}
$$




$$
\begin{aligned}
& +\mathrm{H}(0 ; x)(\mathrm{H}(a, 0,0, b ; x)+\mathrm{H}(0, a, 0, b ; x)+\mathrm{H}(0,0, a, b ; x)) \\
& -\quad(\mathrm{H}(a, 0,0,0, b ; x)+\mathrm{H}(0, a, 0,0, b ; x) \\
& +\mathrm{H}(0,0, a, 0, b ; x)+\mathrm{H}(0,0,0, a, b ; x))
\end{aligned}
$$

In the same way one can use the product identities, Eq.(19) for extracting the terms singular as powers of $\ln (1-x)$, or equivalently of $H(1 ; x)$ according to Eq.(1), around $x=1$ from the H-functions whose leading (or leftmost) indices are equal to 1 . If $a=1$ Eq.(18) can be rewritten as

$$
\begin{aligned}
\mathrm{H}\left(1, m_{1}, \cdots, m_{p} ; x\right) & =\mathrm{H}(1 ; x) \mathrm{H}\left(m_{1}, \cdots, m_{p} ; x\right)-\mathrm{H}\left(m_{1}, 1, m_{2} \cdots, m_{p} ; x\right) \\
& -\mathrm{H}\left(m_{1}, m_{2}, 1, \cdots m_{p} ; x\right)-\cdots-\mathrm{H}\left(m_{1}, \cdots, m_{p-1} m_{p}, 1 ; x\right) .
\end{aligned}
$$

If $m_{1}$ has also the value 1 we can take the second term of the r.h.s. to the left, divide by two and obtain an identity to be used when the first 2 indices are both equal to 1 and so on. Let us show a couple of examples in the case of two indices $a, b$ not equal to 1 :

$$
\begin{aligned}
\mathrm{H}(1,1, a, b ; x)= & \mathrm{H}(1,1 ; x) \mathrm{H}(a, b ; x) \\
- & \mathrm{H}(1 ; x)(\mathrm{H}(a, 1, b ; x)+\mathrm{H}(a, b, 1 ; x)) \\
+ & \mathrm{H}(a, 1,1, b ; x)+\mathrm{H}(a, 1, b, 1 ; x)+\mathrm{H}(a, b, 1,1 ; x), \\
\mathrm{H}(1,1,1, a, b ; x)= & \mathrm{H}(1,1,1 ; x) \mathrm{H}(a, b ; x) \\
- & \mathrm{H}(1,1 ; x)(\mathrm{H}(a, 1, b ; x)+\mathrm{H}(a, b, 1 ; x)) \\
+ & \mathrm{H}(1 ; x)(\mathrm{H}(a, 1,1, b ; x)+\mathrm{H}(a, 1, b, 1 ; x)+\mathrm{H}(a, b, 1,1 ; x)) \\
- & (\mathrm{H}(a, 1,1,1, b ; x)+\mathrm{H}(a, 1,1, b, 1 ; x) \\
& +\mathrm{H}(a, 1, b, 1,1 ; x)+\mathrm{H}(a, b, 1,1,1 ; x)) ;
\end{aligned}
$$

the structure is very much the same as in the equations for extracting the $\ln (x)$ singularities related to the trailing zeroes.

It is to be noted that the two procedures - the "extraction" of leading 1's and trailing 0's - can be combined, to give, for instance

$$
\begin{aligned}
\mathrm{H}(1,1,-1,0 ; x) & =\frac{1}{2} \mathrm{H}(-1 ; x) \mathrm{H}(0 ; x) \mathrm{H}^{2}(1 ; x)-\mathrm{H}(-1,1 ; x) \mathrm{H}(0 ; x) \mathrm{H}(1 ; x) \\
& +\mathrm{H}(-1,1,1 ; x) \mathrm{H}(0 ; x)-\frac{1}{2} \mathrm{H}(0,-1 ; x) \mathrm{H}(1 ; x) \mathrm{H}(1 ; x) \\
& +\mathrm{H}(0,-1,1 ; x) \mathrm{H}(1 ; x)-\mathrm{H}(0,-1,1,1 ; x), \\
\mathrm{H}(1,1,0,0,0 ; x) & =\frac{1}{12} \mathrm{H}^{3}(0 ; x) \mathrm{H}^{2}(1 ; x) \\
& -\mathrm{H}(0,0,0,1 ; x) \mathrm{H}(1 ; x)+\mathrm{H}(0,0,0,1,1 ; x)
\end{aligned}
$$




$$
\begin{aligned}
& +\mathrm{H}(0,0,1 ; x) \mathrm{H}(0 ; x) \mathrm{H}(1 ; x)-\mathrm{H}(0,0,1,1 ; x) \mathrm{H}(0 ; x) \\
& -\frac{1}{2} \mathrm{H}(0,1 ; x) \mathrm{H}^{2}(0 ; x) \mathrm{H}(1 ; x) \\
& +\frac{1}{2} \mathrm{H}(0,1,1 ; x) \mathrm{H}^{2}(0 ; x)
\end{aligned}
$$

Therefore, one can always express a H-function with leading 1's and trailing 0's in terms of products of powers of $H(0 ; x)$ and $H(1 ; x)$, which exhibit the logarithmic singularities in those points, and of other "irreducible" H's, i.e. H's whose first index is not 1 and the last index is not 0 and therefore is finite at both $x=1$ and $x=0$.

We can push further this kind of reduction, by writing all the possible product identities Eq.(19) and the integration by part identities Eq.(17) and using them for expressing as many as possible H's of weight $w$ and "unwanted" indices in terms of products of a "minimal" set of H's of lower weight and "accepted" indices. It is to be noted that the number of the H's in the "minimal" set is fixed, but their choice is not unique, even if the condition of the extraction of the leading 1's and trailing 0 's is imposed. It is easily seen that at weight $w$ the number of relations is nothing but the total number of the different products of H's of lower weight and with total weight $w$. These relations are independent when all H-functions of lower weight belong to their respective "minimal sets". It is to be observed, in any case, that the above "reduction" involves only different rearrangements, without any modification, of the set of indices which appear in the original $\mathrm{H}$,

An explicit calculation gives the set sizes of table 1 .

\begin{tabular}{r|rrr} 
Weight & Full basis & Irreducible set & Minimal set \\
\hline 2 & 9 & 4 & 3 \\
3 & 27 & 12 & 8 \\
4 & 81 & 36 & 18 \\
5 & 243 & 108 & 48 \\
6 & 729 & 324 & 116 \\
7 & 2187 & 972 & 312 \\
8 & 6561 & 2916 & 810
\end{tabular}

Table 1: Sizes of the various bases

The use of the full basis in which each term has only a single H-function gives a unique expression in a rather simple way. This is also the preferred representation when higher weights have to be built up by successive integration. Expressions can also be given in terms of the irreducible set in a relatively easy way. This form is preferred when one has to avoid problems with divergencies. It can also be convenient when establishing identities for related arguments. The use of the minimal set is particularly convenient for the numerical evaluation of the $\mathrm{H}$-functions, when a large number of them has to be evaluated in the same point. It should also be noted that the use of a minimal set is relatively easy for the lower weights (at weight 3 it requires only 4 substitutions) while for higher weights it will much less straightforward. 


\section{Power series expansions}

In general the function $\mathrm{H}_{\vec{m}}(x)$ does not have a regular Taylor series expansion. This is due to the effect that trailing zeroes in the index field may cause powers of $\ln (x)$. Hence the proper expansion is one in terms of both $x$ and $\ln (x)$. Let us first have a look at what happens when there are no logarithms. We will use now the other notation for the indices. In that case we have:

$$
\begin{aligned}
\mathrm{H}_{1}(x) & =\sum_{i=1}^{\infty} \frac{x^{i}}{i} \\
\mathrm{H}_{-1}(x) & =-\sum_{i=1}^{\infty} \frac{(-1)^{i} x^{i}}{i}
\end{aligned}
$$

and assuming that

$$
\mathrm{H}_{\vec{m}}(x)=\sum_{i=1}^{\infty} \frac{\sigma^{i} x^{i}}{i^{a}} S_{\vec{n}}(i)
$$

in which $\sigma= \pm 1$ one can write the relations

$$
\begin{aligned}
\mathrm{H}_{0, \vec{m}}(x) & =\sum_{i=1}^{\infty} \frac{\sigma^{i} x^{i}}{i^{a+1}} S_{\vec{n}}(i) \\
\mathrm{H}_{1, \vec{m}}(x) & =\sum_{i=1}^{\infty} \frac{x^{i}}{i} S_{\sigma a, \vec{n}}(i-1) \\
& =\sum_{i=1}^{\infty} \frac{x^{i}}{i} S_{\sigma a, \vec{n}}(i)-\sum_{i=1}^{\infty} \frac{\sigma^{i} x^{i}}{i^{a+1}} S_{\vec{n}}(i) \\
\mathrm{H}_{-1, \vec{m}}(x) & =-\sum_{i=1}^{\infty} \frac{(-1)^{i} x^{i}}{i} S_{-\sigma a, \vec{n}}(i-1) \\
& =-\sum_{i=1}^{\infty} \frac{(-1)^{i} x^{i}}{i} S_{-\sigma a, \vec{n}}(i)+\sum_{i=1}^{\infty} \frac{\sigma^{i} x^{i}}{i^{a+1}} S_{\vec{n}}(i)
\end{aligned}
$$

At this point one could argue what is the better definition of the nested sums. A definition of the type

$$
Z_{a, \vec{m}}(n)=\sum_{i=1}^{n} \frac{Z_{\vec{m}}(i-1)}{i^{a}}
$$

will give only a single term in the expansion and is favored in the mathematical literature, because there one is mainly concerned with sums to infinity. For finite values of $n$ however this definition has the unelegant aspect that when $\vec{m}$ has $k$ components that are not zero, the value of $Z_{a, \vec{m}}(n)$ is zero for $n \leq k$. We will mostly follow the conventions of ref [6] in which we use the definition:

$$
S_{a, \vec{m}}(n)=\sum_{i=1}^{n} \frac{S_{\vec{m}}(i)}{i^{a}}
$$

\footnotetext{
${ }^{4}$ Because of the linearity of the problem the presence of more than one term, each with a different $S_{\vec{n}}$ would not make much of a difference in the following considerations.
} 
In this notation one has the property $S_{\vec{m}_{k}}(1)=\prod_{i=1}^{k} \sigma_{i}$ with $\sigma_{i}$ being the sign of $m_{i}$. These two notations will be referred to as $Z$-notation and $S$-notation respectively. The conversion from one notation to the other is not really very complicated if one realizes that $\sum_{j=1}^{i-1}=\sum_{j=1}^{i}-\delta_{i j}$. Hence the 'leading' term has the same index field and the correction terms have fewer indices in which some adjacent indices may have been combined. For $k$ nonzero indices there are in total $2^{k-1}-1$ correction terms.

The fact that trailing zeroes in the index field are responsible for powers of $\ln (x)$ can be seen easily now. Because

$$
\frac{1}{k !} \int^{x} d x x^{m} \ln ^{k}(x)=x^{m+1} \sum_{\kappa=0}^{k} \frac{(-1)^{k-\kappa}}{\kappa !} \frac{\ln ^{\kappa}(x)}{(m+1)^{k-\kappa+1}}
$$

we see that once we start with $\mathrm{H}\left(\overrightarrow{0}_{k} ; x\right)$, the subsequent integrations due to other indices (the first of them not being zero of course, and factors $1 /(1 \pm x)$ being expanded in $x)$ that come to the left of the $\overrightarrow{0}_{k}$ will always leave terms with at most $k$ powers of $\ln (x)$ and there will be a term with $k$ of those powers. Hence the trailing zeroes are responsible for powers of $\ln (x)$. Of course the exact dependence of $\ln (x)$ can be derived much easier by applying Eq.(21) repeatedly till all trailing zeroes have been removed. This gives an expansion in terms of powers of $\ln (x)$ and H-functions that are of the type we have just studied and hence can be expanded in $x$. It is however also possible to work one's way through the integrals and the various expansions. This is much more work and leads eventually to the same result. Hence we have omitted this derivation.

If we compare the $\mathrm{H}$-function with the multidimensional polylogarithm in ref [5] we may notice that this function can be rewritten into the following expansion:

$$
\lambda\left(\begin{array}{l}
z_{1} \cdots z_{k} \\
b_{1} \cdots b_{k}
\end{array}\right)=\sum_{\nu_{1}>\nu_{2}>\cdots>\nu_{k}>0}^{\infty} \prod_{j=1}^{k} \frac{b_{j-1}^{\nu_{j}}}{\nu_{j}^{z_{j}} b_{j}^{\nu_{j}}}
$$

with $b_{0}=1$. These functions do not contain powers of $\ln \left(b_{i}\right)$ and hence they cannot represent all $\mathrm{H}$-functions. If we restrict ourselves to $\mathrm{H}$-functions without trailing zeroes one can write the terms in the expansion of these $\mathrm{H}$-functions as

$$
\sum_{\nu_{1}>\nu_{2}>\cdots>\nu_{k}>0}^{\infty} x^{\nu_{1}} \prod_{j=1}^{k} \frac{\sigma_{j}^{\nu_{j}}}{\nu_{j}^{z_{j}}}
$$

if we use $Z$-sums and

$$
\sum_{\nu_{1} \geq \nu_{2} \geq \cdots \geq \nu_{k} \geq 1}^{\infty} x^{\nu_{1}} \prod_{j=1}^{k} \frac{\sigma_{j}^{\nu_{j}}}{\nu_{j}^{s_{j}}}
$$

if we use $S$-sums. Hence it is clear that the $\mathrm{H}$-functions without trailing zeroes are special cases of the multidimensional polylogarithms with $b_{i}= \pm 1 / x$. For the computation of Feynman diagrams we do however need the H-functions with trailing zeroes because of the presence of the logarithms (see for instance ref [10]). 
There is another interesting observation in the expansion. Considering that the expansion of an $\mathrm{H}$-function with no trailing zeroes gives terms of the type

$$
\sum_{x=1}^{\infty} x^{i} \frac{\sigma^{i} S_{\vec{m}}(i)}{i^{a}}
$$

one can introduce another sum by dividing by either $1+x$ or $1-x$ and obtain:

$$
\begin{aligned}
\sum_{x=1}^{\infty} x^{i} \frac{\sigma^{i} S_{\vec{m}}(i)}{i^{a}} & =(1-x) \sum_{x=1}^{\infty} x^{i} S_{\sigma a, \vec{m}}(i) \\
& =(1+x) \sum_{x=1}^{\infty} x^{i}(-1)^{i} S_{-\sigma a, \vec{m}}(i)
\end{aligned}
$$

At times this notation is more convenient. One should however remember that this notation breaks down at either $x=1$ or at $x=-1$, depending on the particular form used.

Finally we notice that for $x=1$ we have that

$$
\sum_{x=1}^{\infty} x^{i} \frac{\sigma^{i} S_{\vec{m}}(i)}{i^{a}} \rightarrow S_{\sigma a, \vec{m}}(\infty)
$$

and hence the values of the $\mathrm{H}$-functions in $x=1$ are related to the values of the $S$-sums in infinity. The trailing zeroes do not cause essential problems because when those functions are first written in terms of powers of $\ln (x)$ these logarithms vanish in $x=1$ and we keep only the terms with $\mathrm{H}$-functions without trailing zeroes. For the numerical evaluation of these objects one can use the algorithms of ref [5] that relate them effectively to combinations of $\mathrm{H}$-functions in $x=1 / 2$ after the appropriate conversions. This is particularly interesting for the higher weights because up to weight 7,8 or 9 it is still possible to obtain expressions in terms of a very small number of constants (see ref [9] and [6]), but beyond these weights this becomes too time consuming while an expansion in $x=1 / 2$ is sufficiently fast for nearly all numerical applications, provided that only a limited number of them is needed.

\section{The algebra}

The harmonic sums form an algebra [6] in which the product of two sums with the same argument and having weights $w_{1}$ and $w_{2}$ respectively can be written as a sum of terms, each with a single sum of weight $w_{1}+w_{2}$. There are two sets of algebraic relations: the relations based on the shuffle algebra which hold for all values of the argument, and the relations based on the triangle theorem of ref [6] which hold only for values in infinity, provided that not both harmonic sums are divergent. For the H-functions we have the general product formula based on Eq.(19). This formula is related to the algebra of the harmonic sums, because the harmonic polylogarithms can be expressed in

\footnotetext{
${ }^{5}$ Thus far the only known exact method to do this involves solving simultaneously for all $23^{w-1} \mathrm{H}$-functions in $x=1$. See also a previous footnote.
} 
terms of series expansions in which the coefficients are harmonic sums: assume for the moment that neither $\vec{m}$ nor $\vec{n}$ have trailing zeroes. In that case we derive:

$$
\mathrm{H}_{a, \vec{m}_{p}}(x) \mathrm{H}_{\vec{n}_{q}}(x)=\frac{1}{1-x} \sum_{i=1}^{\infty} \frac{S_{\vec{m}_{p}}(i) x^{i}}{i^{a}} \sum_{j=1}^{\infty} S_{\vec{n}_{q}}(j) x^{j}
$$

in which one of the two powers of $1 /(1-x)$ has been absorbed in the sum over $i$. By combining the powers of $x$ this formula can be rewritten as

$$
\mathrm{H}_{a, \vec{m}_{p}}(x) \mathrm{H}_{\vec{n}_{q}}(x)=\frac{1}{1-x} \sum_{i=1}^{\infty} x^{i} \sum_{j=1}^{i} \frac{S_{\vec{m}}(j) S_{\vec{n}}(i-j)}{j^{a}} .
$$

Note that the inner sum can be done and gives a set of terms that are all single $S$ functions, even though the expression may not be very compact. It is called a triangle sum and an algorithm for it is given in one of the appendices of ref [6]. It is also available as a procedure in the language of FORM [7]. As a result one obtains an expression which can be resummed and gives terms with single H-functions.

For H-functions in $x=1$ we have seen that they can be directly expressed in terms of harmonic sums in infinity. Therefore the general algebraic rules for those sums that are based on the shuffle algebra for harmonic sums can be applied. Hence we see a duality here: the general rules for the $\mathrm{H}$-functions correspond to the special triangle rules for the harmonic sums, and the special rules for the H-functions in $x=1$ correspond to the general shuffle rules for the harmonic sums.

There is one complicating factor when values in $x=1$ are considered. Let us start with assuming that the basic divergence $\mathrm{H}(1 ; 1)$ can be used as a symbol. In the case of a 'proper' limit procedure such things can be done and after the divergences cancel the finite result should be correct. This is called regularization. The general algebraic relations are based on the triangle sums, rather than on the shuffle algebra, and the triangle sums are not correct when both objects are divergent. The subleading terms will be incorrect. This can be illustrated easily:

$$
\begin{aligned}
\mathrm{H}_{1}(x) & =\sum_{i=1}^{\infty} \frac{x^{i}}{i} \\
\left(\mathrm{H}_{1}(x)\right)^{2} & =2 \mathrm{H}_{1,1}(x) \\
& =2 \sum_{i=1}^{\infty} x^{i}\left(\frac{S_{1}(i)}{i}-\frac{1}{i^{2}}\right) \\
\mathrm{H}_{1}(1) & =\lim _{x \rightarrow 1} \sum_{i=1}^{\infty} \frac{x^{i}}{i} \\
& =S_{1}(\infty) \\
\mathrm{H}_{1,1}(1) & =\lim _{x \rightarrow 1} 2 \sum_{i=1}^{\infty} x^{i}\left(\frac{S_{1}(i)}{i}-\frac{1}{i^{2}}\right) \\
& \left.=2 S_{1,1}(\infty)-2 S_{2}(\infty)\right) \\
& \left.=\left(S_{1}(\infty)\right)^{2}-S_{2}(\infty)\right)
\end{aligned}
$$


and we see that

$$
\left(\lim _{x \rightarrow 1} \mathrm{H}_{1}(x)\right)^{2} \neq \lim _{x \rightarrow 1}\left(\mathrm{H}_{1}(x)\right)^{2} .
$$

The solution to this problem is to be found in $S$-space. There it is possible to regularize the infinite sums in a consistent way by replacing the sum to infinity by a sum to $M$ with $M$ very large but finite, then one can have the divergences cancel and finally take the limit $M \rightarrow \infty$. This does not correspond to anything one can do in $x$-space. Because the triangle theorem does not hold for two $S$-sums that are divergent, one cannot apply the regular algebraic relation for H-functions that are both divergent in $x=1$. Hence the proper algebraic relations at $x=1$ have to be derived by means of the shuffle algebra which holds for all $S$-sums:

$$
\begin{aligned}
\left(\lim _{x \rightarrow 1} \mathrm{H}_{1}(x)\right)^{2} & =\left(S_{1}(\infty)\right)^{2} \\
& =2 S_{1,1}(\infty)-S_{2}(\infty) \\
& =2 \lim _{x \rightarrow 1} \mathrm{H}_{1,1}(x)+\lim _{x \rightarrow 1} \mathrm{H}_{2}(x)
\end{aligned}
$$

This way is consistent and will allow us to define the Mellin transform properly in one of the next sections. It involves the use of values in $x=1$.

Because of the use of different algebraic relations for $x \neq 1$ and $x=1$, it may happen that expressions look rather complicated, but the various algebraic relations between H-functions in $x=1$ could simplify the expressions considerably. However at the moment there is no known systematic method to apply these relations in such a way that one does not have to solve for all values in $x=1$ first. This way all such objects can be expressed in a minimal independent set of objects. Unfortunately there are very many of these objects for a given weight $w\left(23^{w-1}\right)$ and even more relations and hence it is a formidable task to determine all values at $x=1$ in terms of a minimal set of constants when the weight is large. If the final answer is supposed to be finite one can however extract the powers of the basic divergences (they correspond to leading indices that are 1) and hence still obtain a finite answer that can be evaluated numerically. The coefficients of the divergences can be checked to be zero numerically as well.

\section{Identities between H-functions of related arguments.}

In this section we will look at the identities which can be established for suitable changes of the argument. The common feature is that any H-function of weight $w$ and argument $x$ can be expressed as an homogeneous expression of the same weight $w$, involving either $\mathrm{H}$-functions depending on a same argument, say $t$, related to $x$ by the considered change, or constants corresponding to $\mathrm{H}$-functions of special constant values of the arguments (typically 1 ).

The simplest change of the argument is the change $x \rightarrow-x$. We have seen its effect already in Eq.(15).

Next is the relation between H-functions of $x^{2}$ and of $x$. Because $1+x^{2}$ is not a particularly interesting object we will have to exclude indices equal to -1 in the H-functions of $x^{2}$. Restricting 
the indices to only 1 and 0 , we can proceed recursively on the weight. For weight 1 we have from Eq.(11):

$$
\begin{aligned}
& \mathrm{H}\left(0 ; x^{2}\right)=2 \mathrm{H}(0 ; x) \\
& \mathrm{H}\left(1 ; x^{2}\right)=\mathrm{H}(1 ; x)-\mathrm{H}(-1 ; x)
\end{aligned}
$$

so that the H's of argument $x^{2}$ are expressed in terms of H's of argument $x$, as required.

For $w>1$, if $\vec{m}_{w}=\overrightarrow{0}_{w}$,

$$
\mathrm{H}\left(\overrightarrow{0}_{w} ; x^{2}\right)=2^{w} \mathrm{H}\left(\overrightarrow{0}_{w} ; x\right) ;
$$

otherwise, if $\vec{m}_{w}=\left(a, \vec{m}_{w-1}\right)$ for the two cases $a=0$ and $a=1$ we have, by using the change of variable $x^{\prime}=t^{\prime 2}$

$$
\begin{aligned}
\mathrm{H}\left(0, \vec{m}_{w-1} ; x^{2}\right) & =\int_{0}^{x^{2}} \frac{d x^{\prime}}{x^{\prime}} \mathrm{H}\left(\vec{m}_{w-1} ; x^{\prime}\right) \\
& =2 \int_{0}^{x} \frac{d t^{\prime}}{t^{\prime}} \mathrm{H}\left(\vec{m}_{w-1} ; t^{\prime 2}\right) \\
\mathrm{H}\left(1, \vec{m}_{w-1} ; x^{2}\right) & =\int_{0}^{x^{2}} \frac{d x^{\prime}}{1-x^{\prime}} \mathrm{H}\left(\vec{m}_{w-1} ; x^{\prime}\right) \\
& =\int_{0}^{x} d t^{\prime}\left(\frac{1}{1-t^{\prime}}-\frac{1}{1+t^{\prime}}\right) \mathrm{H}\left(\vec{m}_{w-1} ; t^{\prime 2}\right) .
\end{aligned}
$$

The expression of the $\mathrm{H}\left(\vec{m}_{w-1} ; t^{\prime 2}\right)$ in terms of H's of the same weight and argument $t^{\prime}$ is supposedly known (as we proceed recursively on the weight $w$ ); by substituting such expression and then using the very definition Eq.(6) all the required $x^{2} \rightarrow x$ identities are obtained. An example of weight $w=2$ is

$$
\begin{aligned}
\mathrm{H}\left(0,1 ; x^{2}\right) & =2 \int_{0}^{x} \frac{d t^{\prime}}{t^{\prime}} \mathrm{H}\left(1 ; t^{\prime 2}\right) \\
& =2 \int_{0}^{x} \frac{d t^{\prime}}{t^{\prime}}\left(\mathrm{H}\left(1 ; t^{\prime}\right)-\mathrm{H}\left(-1 ; t^{\prime}\right)\right) \\
& =2 \mathrm{H}(0,1 ; x)-2 \mathrm{H}(0,-1 ; x)
\end{aligned}
$$

and Eq.15) leads to the well known relation $\operatorname{Li}_{2}\left(x^{2}\right)=2 \operatorname{Li}_{2}(x)+2 \operatorname{Li}_{2}(-x)$. We can observe here that a limited set of $x^{2} \rightarrow x$ identities could be written only for the Nielsen's polylogarithms corresponding to the $\mathrm{H}_{n}(x)$ in the notation of Eq.(14), while for the hpl's the set is wider; as an example, one can derive for $w=3$ :

$$
\mathrm{H}\left(1,0,1 ; x^{2}\right)=2(\mathrm{H}(1,0,1 ; x)-\mathrm{H}(-1,0,1 ; x)-\mathrm{H}(1,0,-1 ; x)+\mathrm{H}(-1,0,-1 ; x))
$$

The next transformation of the argument we consider is $x \rightarrow 1-x$ which applies again to a smaller set of Nielsen's polylogarithms. Like the previous transformation it is of interest only when there are no negative indices $(1+x \rightarrow 2-x$ is not something we can work with). Proceeding 
recursively on $w$, as before, for $w=1$ we have

$$
\begin{aligned}
& \mathrm{H}(0 ; 1-x)=-\mathrm{H}(1 ; x) \\
& \mathrm{H}(1 ; 1-x)=-\mathrm{H}(0 ; x) .
\end{aligned}
$$

The extension to higher weights requires a minimum of care. $\mathrm{H}\left(a, \vec{m}_{w-1} ; 1-x\right)$ of weight $w>1$, with the first index $a$ equal to 0 or to 1 is the generic function. As discussed in Section 3 , if $a=1$ the function can be expressed in terms of a reduced set of functions, where the leading index 1 is carried only by $\mathrm{H}(1 ; 1-x)$, for which Eq. (48) holds; therefore, only the case in which the first index $a$ is 0 is to be considered. In that case, the change of variable $x^{\prime}=1-t^{\prime}$ gives

$$
\begin{aligned}
\mathrm{H}\left(0, \vec{m}_{w-1} ; 1-x\right) & =\int_{0}^{1-x} \frac{d x^{\prime}}{x^{\prime}} \mathrm{H}\left(\vec{m}_{w-1} ; x^{\prime}\right) \\
& =\int_{0}^{1} \frac{d x^{\prime}}{x^{\prime}} \mathrm{H}\left(\vec{m}_{w-1} ; x^{\prime}\right)-\int_{1-x}^{1} \frac{d x^{\prime}}{x^{\prime}} \mathrm{H}\left(\vec{m}_{w-1} ; x^{\prime}\right) \\
& =\mathrm{H}\left(0, \vec{m}_{w-1} ; 1\right)-\int_{0}^{x} \frac{d t^{\prime}}{1-t^{\prime}} \mathrm{H}\left(\vec{m}_{w-1} ; 1-t^{\prime}\right),
\end{aligned}
$$

where the constant $\mathrm{H}\left(0, \vec{m}_{w-1} ; 1\right)$ is finite (it can be observed here that if the first index is 1 one runs into the problem that $\mathrm{H}\left(1, \vec{m}_{w-1} ; 1\right)$ could be divergent). In the general case $\mathrm{H}\left(\vec{m}_{w-1} ; 1-t^{\prime}\right)$ will not be irreducible. We can express it in terms of the H's of an irreducible set of weight $w-1$, use the supposedly known $x=1-t$ identities of weight $w-1$ and finally obtain the required weight $w$ identity by using the definition Eq.(6). As an example we have at weight 4

$$
\begin{aligned}
\mathrm{H}(0,0,1,1 ; 1-x) & =\mathrm{H}(0,0,1,1 ; 1)-\mathrm{H}(1 ; x) \mathrm{H}(0,1,1 ; 1)+\mathrm{H}(1,1,0,0 ; x) \\
& =\mathrm{H}(0,0,1,1 ; 1)-\mathrm{H}(0,1,1 ; 1) \mathrm{H}(1 ; x)-\mathrm{H}(0,0,1,1 ; x) \\
& +\frac{1}{4} \mathrm{H}^{2}(0 ; x) \mathrm{H}^{2}(1 ; x)-\mathrm{H}(0,1 ; x) \mathrm{H}(0 ; x) \mathrm{H}(1 ; x) \\
& +\mathrm{H}(0,0,1 ; x) \mathrm{H}(1 ; x)+\mathrm{H}(0,1,1 ; x) \mathrm{H}(0 ; x)
\end{aligned}
$$

A transformation which applies to all the Nielsen's polylogarithms, Eq.(16) is

$$
x=1 / y ; \quad y=1 / x ;
$$

it will be shown that it applies as well to all the H-functions. Before continuing, let us recall that the Nielsen's polylogarithms have a (logarithmic) branch point at $x=1$, but are otherwise analytic for smaller values of $x$, including all the negative real axis; for studying the transformation Eq.(51) it can be therefore convenient to establish the identities for negative values of $x$, and then continue analytically to positive values. The analytic properties of the H-functions are more complicated. First of all, if the rightmost index is equal to 0 , they have a branch point at $x=0$; that is not a problem, as we have already seen that we can express any $\mathrm{H}$-function in terms of the functions of a reduced set where the trailing index 0 is carried only by powers of $H(0 ; x)=\ln x$, whose analytic properties are well known. If the rightmost index is not 0 and all the indices are in general equal to 1 or 0 , the H-functions have the same analytic properties as the Nielsen's polylogarithms; but 
if some of indices are equal to -1 , a branch cut at $x=-1$ appears. Therefore, in the general case when indices equal to -1 are also present (and that is the case even of the reduced and minimal sets, see Section 3), there is no advantage in considering negative values of $x$, so that we will start from the beginning with an argument equal to $x+i \epsilon$, where $x$ is real and satisfies the constraints $0 \leq x \leq 1$, while $\epsilon$ is positive and infinitesimally small; correspondingly,

$$
y=1 / x-i \epsilon,
$$

i.e. the real part of $y$ is also positive, but $y \geq 1$, while its infinitesimal imaginary part is negative.

As in the previous cases, we will proceed by induction on the weight $w$ of the H-functions. At $w=1$ we have

$$
\begin{aligned}
\mathrm{H}(0 ; y) & =-\mathrm{H}(0 ; x), \\
\mathrm{H}(1 ; y) & =\mathrm{H}(1 ; x)+\mathrm{H}(0 ; x)-i \pi, \\
\mathrm{H}(-1 ; y) & =\mathrm{H}(-1 ; x)-\mathrm{H}(0 ; x) ;
\end{aligned}
$$

the constant $\pi$ has appeared; it must be given weight 1 , so that all the formulas will remain homogeneous of the same weight. When continuing the above equations to negative values of $x$, in the interval $-1 \geq x \geq 0, H(0 ; x)=\ln (x+i \epsilon)$ will develop a positive imaginary part; in particular, one has

$$
\mathrm{H}(0 ;-1)=i \pi,
$$

so that $\mathrm{H}(1 ;-1)$ takes the real value $-\ln 2$, as expected.

For $w>1, \vec{m}_{w}=\left(a, \vec{m}_{w-1}\right)$, we can proceed by induction, along the following lines

$$
\begin{aligned}
\mathrm{H}\left(\vec{m}_{w} ; y\right) & =\int_{0}^{y} d y^{\prime} f\left(a ; y^{\prime}\right) \mathrm{H}\left(\vec{m}_{w-1} ; y^{\prime}\right) \\
& =\int_{0}^{1} d y^{\prime} f\left(a ; y^{\prime}\right) \mathrm{H}\left(\vec{m}_{w-1} ; y^{\prime}\right)+\int_{1}^{y} d y^{\prime} f\left(a ; y^{\prime}\right) \mathrm{H}\left(\vec{m}_{w-1} ; y^{\prime}\right) \\
& =\mathrm{H}\left(\vec{m}_{w} ; 1\right)+\int_{x}^{1} \frac{d x^{\prime}}{x^{\prime 2}} f\left(a, \frac{1}{x^{\prime}}\right) \mathrm{H}\left(\vec{m}_{w-1} ; \frac{1}{x^{\prime}}\right) .
\end{aligned}
$$

It is to be noted that one can assume that the first index $a$ is different from 1 ; indeed, as seen in Section 3 any $\mathrm{H}$-function of the form $\mathrm{H}\left(1, \vec{m}_{w-1} ; y\right)$ can be expressed in terms of a reduced set of functions, where the leading index 1 is carried only by powers of $\mathrm{H}(1 ; y)$, whose transformation is given by Eq.(53). For a different from $1, \mathrm{H}\left(\vec{m}_{w} ; 1\right)$ is a finite constant and the above formulae are meaningful. One further finds

$$
\begin{aligned}
\int \frac{d x^{\prime}}{x^{\prime 2}} f\left(0 ; \frac{1}{x^{\prime}}\right) & =+\int d x^{\prime} \frac{1}{x^{\prime}} \\
\int \frac{d x^{\prime}}{x^{\prime 2}} f\left(-1 ; \frac{1}{x^{\prime}}\right) & =+\int d x^{\prime}\left(\frac{1}{x^{\prime}}-\frac{1}{1+x^{\prime}}\right) ;
\end{aligned}
$$

substituting in the r.h.s. of Eq.(55) the identities (of weight $w-1$, and therefore known in an approach by induction) which express $\mathrm{H}\left(\vec{m}_{w-1} ; y^{\prime}=1 / x^{\prime}\right)$ in terms of $\mathrm{H}\left(\vec{m}^{\prime}{ }_{w-1} ; x^{\prime}\right)$, one obtains a 
combination of terms of the kind

$$
\int_{x}^{1} d x^{\prime} f\left(a ; x^{\prime}\right) \mathrm{H}_{{\overrightarrow{m^{\prime}}}_{w-1}}\left(x^{\prime}\right)=\mathrm{H}\left(a,{\overrightarrow{m^{\prime}}}_{w-1} ; 1\right)-\mathrm{H}\left(a,{\overrightarrow{m^{\prime}}}_{w-1} ; x\right)
$$

and the identities of weight $w$ are established. As an example, we give the $w=3$ identity

$$
\begin{aligned}
\mathrm{H}\left(0,-1,1 ; \frac{1}{x}-\right. & i \epsilon)=-\mathrm{H}(0,-1,1 ; x)+2 \mathrm{H}(0,-1,1 ; 1) \\
& +2 \mathrm{H}(0,0,-1 ; x)-2 \mathrm{H}(0,0,-1 ; 1)+\mathrm{H}(0,0,1 ; x)-\mathrm{H}(0,0,1 ; 1) \\
& -(\mathrm{H}(0,-1 ; x)+\mathrm{H}(0,-1 ; 1)+\mathrm{H}(0,1 ; 1)) \mathrm{H}(0 ; x)+\frac{1}{6} \mathrm{H}^{3}(0 ; x) \\
& -i \pi\left(\mathrm{H}(-1 ; 1) \mathrm{H}(0 ; x)+\frac{1}{2} \mathrm{H}^{2}(0 ; x)-\mathrm{H}(0,-1 ; x)+H(0,-1 ; 1)\right) .
\end{aligned}
$$

Another important set of identities, which is however valid for any set of indices and has no counterpart within the Nielsen's polylogarithms, applies to arguments $x$ and $t$ related by the transformation

$$
x=\frac{1-t}{1+t},
$$

whose inverse is again

$$
t=\frac{1-x}{1+x} .
$$

Even in that case, it turns out that any H-function of weight $w$ and argument $x$ can be expressed as a homogeneous expression of weight $w$, involving $\mathrm{H}$-functions of argument $t$, related to $x$ by Eq.(57), as well as constants corresponding to H-functions of argument 1 . The proof is, again, by induction on the weight. If $w=1$, from the very definition Eq.(1) one immediately finds

$$
\begin{aligned}
\mathrm{H}(0 ; x) & =-\mathrm{H}(1 ; t)-\mathrm{H}(-1 ; t), \\
\mathrm{H}(1 ; x) & =-\mathrm{H}(0 ; t)-\mathrm{H}(-1 ; 1)+\mathrm{H}(-1 ; t), \\
\mathrm{H}(-1 ; x) & =\mathrm{H}(-1 ; t)-\mathrm{H}(-1 ; 1) .
\end{aligned}
$$

For $w>1$ and $\vec{m}_{w}=\overrightarrow{0}_{w}$ the result is trivially true, as can be verified by inspection; the same is true also for $\vec{m}_{w}=\overrightarrow{1}_{w}$ and $\vec{m}_{w}=\overrightarrow{-1_{w}}$. In the more general case, write $\vec{m}_{w}=\left(a, \vec{m}_{w-1}\right)$; where the index $a$ takes the values $0,1,-1$. As discussed in Section 3, and already recalled for the $x \rightarrow 1-x$ identities, if $a=1$ the function can be expressed in terms of a reduced set of functions, where the leading index 1 is carried only by $\mathrm{H}(1 ; x)$, for which Eq.(59) holds. In the other two cases $a=0,-1$ the change of variable

$$
x^{\prime}=\frac{1-t^{\prime}}{1+t^{\prime}}
$$

gives

$$
\mathrm{H}\left(0, \vec{m}_{w-1} ; x\right)=\int_{0}^{x} \frac{d x^{\prime}}{x^{\prime}} \mathrm{H}\left(\vec{m}_{w-1} ; x^{\prime}\right)
$$




$$
\begin{aligned}
& =\mathrm{H}\left(0, \vec{m}_{w-1} ; 1\right)-\int_{x}^{1} \frac{d x^{\prime}}{x^{\prime}} \mathrm{H}\left(\vec{m}_{w-1} ; x^{\prime}\right) \\
& =\mathrm{H}\left(0, \vec{m}_{w-1} ; 1\right)-\int_{0}^{t} d t^{\prime}\left(\frac{1}{1-t^{\prime}}+\frac{1}{1+t^{\prime}}\right) \mathrm{H}\left(\vec{m}_{w-1} ; \frac{1-t^{\prime}}{1+t^{\prime}}\right), \\
\mathrm{H}\left(-1, \vec{m}_{w-1} ; x\right) & =\int_{0}^{x} \frac{d x^{\prime}}{1+x^{\prime}} \mathrm{H}\left(\vec{m}_{w-1} ; x^{\prime}\right) \\
& =\mathrm{H}\left(-1, \vec{m}_{w-1} ; 1\right)-\int_{0}^{t} d t^{\prime} \frac{1}{1+t^{\prime}} \mathrm{H}\left(\vec{m}_{w-1} ; \frac{1-t^{\prime}}{1+t^{\prime}}\right) .
\end{aligned}
$$

At this point, one can substitute the relations already found to be valid at weight $w-1$, for expressing the functions $\mathrm{H}\left(\vec{m}_{w-1} ;\left(1-t^{\prime}\right) /\left(1+t^{\prime}\right)\right)$ in terms of H's of weight $w-1$ and argument $t^{\prime}$, and then perform the last integration in $t^{\prime}$ according to the definition Eq.(6).

As an example, we give the following $w=3$ identity

$$
\begin{gathered}
\mathrm{H}(-1,-1,1 ; x)=-\mathrm{H}(0,-1,-1 ; t)+\mathrm{H}(-1,-1,1 ; 1) \\
+\mathrm{H}(0,-1 ; t) \mathrm{H}(-1 ; t)+\frac{1}{6} \mathrm{H}^{3}(-1 ; t)-\frac{1}{2} \mathrm{H}^{2}(-1 ; t) \mathrm{H}(0 ; t) \\
-\frac{1}{2} \mathrm{H}(-1 ; 1) \mathrm{H}^{2}(-1 ; t)-\mathrm{H}(-1,1 ; 1) \mathrm{H}(-1 ; t) .
\end{gathered}
$$

\section{$7 \quad$ Identities between H's and related functions.}

Let us introduce a related set of functions $\mathrm{G}\left(\vec{m}_{w} ; x\right)$, where $\vec{m}_{w}$ has almost the same meaning as for the H's, but the first index $m_{w}$ is always equal to 1, i.e. $\vec{m}=\left(1, \vec{m}_{w-1}\right)$, through the definitions

$$
\mathrm{G}(1 ; x)=-\int_{0}^{1} \frac{d t}{t-1 / x}
$$

for $w=1$ and

$$
\mathrm{G}\left(1, \vec{m}_{w-1} ; x\right)=-\int_{0}^{1} \frac{d t}{t-1 / x} \mathrm{H}\left(\vec{m}_{w-1} ; t\right)
$$

for $w>1$.

The $\mathrm{G}\left(\vec{m}_{w} ; x\right)$ are nothing but homogeneous combination of $\mathrm{H}$-functions of weight $w$. As by now usual, we will show it proceeding by induction on $w$. For $w=1$, by performing explicitly the elementary integration we obtain from Eq.(62)

$$
\mathrm{G}(1 ; x)=\mathrm{H}(1 ; x)
$$

Next, assume that the identities are established for $w$; put $\vec{m}=\left(a, \vec{m}_{w-1}\right)$, and consider the functions of weight $w+1$ given by

$$
\mathrm{G}\left(1, a, \vec{m}_{w-1} ; x\right)=-\int_{0}^{1} \frac{d t}{t-1 / x} \mathrm{H}\left(a, \vec{m}_{w-1} ; t\right) .
$$


One can differentiate with respect to $x$, then integrate by parts in $t$, using of course Eq.(63) when relevant; considering for instance the case $a=-1$ one obtains

$$
\begin{aligned}
\frac{\partial}{\partial x} \mathrm{G}\left(1,-1, \vec{m}_{w-1} ; x\right) & =[f(-1, x)-f(0, x)] \mathrm{G}\left(1, \vec{m}_{w-1} ; x\right) \\
& +[f(-1, x)+f(1, x)] \mathrm{H}\left(-1, \vec{m}_{w-1} ; 1\right)
\end{aligned}
$$

Similarly, one has

$$
\begin{aligned}
\frac{\partial}{\partial x} \mathrm{G}\left(1,0, \vec{m}_{w-1} ; x\right) & =-f(0, x) \mathrm{G}\left(1, \vec{m}_{w-1} ; x\right) \\
& +f(-1, x) \mathrm{H}\left(0, \vec{m}_{w-1} ; 1\right) \\
\frac{\partial}{\partial x} \mathrm{G}\left(1,1, \vec{m}_{w-1} ; x\right) & =[f(0, x)+f(1, x)] \mathrm{G}\left(1, \vec{m}_{w-1} ; x\right) .
\end{aligned}
$$

One can substitute the already obtained identities expressing $\mathrm{G}\left(1, \vec{m}_{w-1} ; x\right)$ in terms of H's of weight $w$ and then integrate in $x$ between 0 and $x$ by using the very definition Eq.(6) (according to Eq. (63) the G-functions vanish at $x=0$ ). The required identities of weight $w+1$ are then established. As an example, we give one of the identities of weight $w=4$

$$
\begin{aligned}
\mathrm{G}(1,0,-1,1 ; x) & =-\mathrm{H}(0,-1,0,1 ; x)-\mathrm{H}(0,-1,1,1 ; x) \\
& +\mathrm{H}(0,0,0,1 ; x)+\mathrm{H}(0,0,1,1 ; x) \\
& -\mathrm{H}(-1,1 ; 1) \mathrm{H}(0,-1 ; x)-\mathrm{H}(-1,1 ; 1) \mathrm{H}(0,1 ; x) \\
& +\mathrm{H}(0,-1,1 ; 1) \mathrm{H}(1 ; x) .
\end{aligned}
$$

In the same way one can work out the similar identities existing for several related classes of functions such as, for instance,

$$
-\int_{0}^{1} \frac{d t}{t-1 / x} \mathrm{H}(\vec{a} ; t) \mathrm{H}(\vec{b} ; x t)
$$

or

$$
\int_{0}^{1} d t f(a, t) \mathrm{H}(\vec{a} ; t) \mathrm{H}(\vec{b} ; x t)
$$

\section{Special values of the H's and their numerical evaluation.}

It is known that the Nielsen's polylogarithms for the special values of the arguments equal to $+1,-1$ and $1 / 2$ can be expressed in terms of a few mathematical constants, typically Riemann $\zeta$-functions of integer arguments; the representations which they provide for those constants as definite integrals can be manipulated by means of integration by parts, changes of variables and the like providing the analytic values of a number of definite integrals of special interest. The same applies, and in much more systematic way, to the H-functions, thanks to the greater and wider sets of identities which they satisfy. 
In the case of the H's, it is not necessary to consider as independent the values corresponding to the argument equal to -1 ; indeed, one can always express any $\mathrm{H}$-function in terms of the reduced set of functions in which trailing indices equal to 0 are missing, so that by using Eq.(15) one can replace a value at $x=-1$ with the value at $x=1$ of a related function. In analogy with the Nielsen's polylogarithms case, it is convenient to consider also the values at $x=1 / 2$ of the functions whose indices are equal to 0 or 1 (i.e. when the index -1 is missing).

More specifically, one can consider:

- the $x^{2} \rightarrow x$ identities, Eq.s(42-47), for $x=1$;

- the $1-x \rightarrow x$ identities, Eq.s(48 50 ). They can be used at $x=1 / 2$, providing a first set of identities for the values at $x=1 / 2$, but also at $x=-1$; in the second case, one gets values at $x=2$, which are converted into values at $x=1 / 2$ by using the $x \rightarrow 1 / x$ identities, Eq.s(51-56), as well as values at $x=-1$, which are converted into values at $x=1$ by Eq.(15);

- the just recalled $x \rightarrow 1 / x$ identities, Eq.s(51 56$)$, at $x=1$ and $x=-1$, followed by the usual conversion to $x=1$ through Eq.(15);

- the $x \rightarrow(1-t) /(1+t)$ identities, Eq.s(57- 61), at $x=0$ corresponding to $t=1$ (they are automatically satisfied, by construction, at $x=1, t=0)$;

- one more set of identities is obtained by writing the identities between G-functions and Hfunctions, discussed in Section 8 , at the special value $x=-1$, by using the relation, which follows from the definition Eq.(63)

$$
\mathrm{G}(1, \vec{m} ;-1)=\mathrm{H}(-1, \vec{m} ; 1)
$$

and converting once more the values at $x=-1$ of the H's into values at $x=1$ by means of Eq.(15).

The set of relations obtained in that way is highly redundant; it has been checked explicitly that they generate the table of the $w=4$ definite integrals given in Appendix B of the second reference of [2]. It has not yet been investigated whether they are sufficient, by themselves, to generate also the tables of higher weights obtained in [6].

Another powerful method to obtain the values at $x=1 / 2$ when there are no negative indices is by considering the transformation $x \rightarrow z /(1+z)$, which corresponds to a suitable combination of the transformations $x \rightarrow 1 / x$ and $x \rightarrow(1-x)$. Using the same techniques as in the section on related arguments, all these objects are directly expressed in terms of H-functions in $x=1$. Such expressions can then be used in reverse to obtain the numerical values of the 'independent constants' that occur in the expressions for the H-functions at $x=1$. As an example we have

$$
\operatorname{Li}_{3}\left(\frac{1}{2}\right)=\frac{7}{8} \zeta_{3}-\frac{1}{2} \zeta_{2} \ln (2)+\frac{1}{6} \ln ^{3}(2)
$$


which is of course well known. We have also

$$
\mathrm{H}_{2,1}\left(\frac{1}{2}\right)=\frac{1}{8} \zeta_{3}-\frac{1}{6} \ln ^{3}(2)
$$

Both relations provide a power series for the evaluation of $\zeta_{3}$. The method gives also an expression of $s_{6}=S_{-5,-1}(\infty)$ in terms of $\mathrm{H}_{5,1}(1 / 2), \mathrm{H}_{6}(1 / 2)$ and combinations of constants of a lower weight. Similar dependencies can be derived for the higher weight constants.

Let us finish with a few remarks on the numerical evaluation of the H's for arbitrary values of $x$. According to the discussion of Section 3, it is sufficient to restrict ourselves to the H's either of the reduced set or of a minimal set, as all the others can be obtained from them as suitable combinations. The H's of such a set have no trailing indices equal to 0 , so that they can be expanded in series of $x$ around $x=0$. For small values of $x$ the series will be rapidly convergent, but the convergence will slow down approaching the cuts at $x= \pm 1$. But for $x$ approaching 1 we can use the transformation Eq.(57), so that the corresponding $t=(1-x) /(1+x)$ will fall in the region near 0 and the expansion in $t$ will be rapidly converging.

More exactly, the equation

$$
r=\frac{1-r}{1+r}
$$

has the two solutions $r=-1-\sqrt{2}$ and $r=-1+\sqrt{2}$. Therefore, we can use the expansion around $x=0$ in the interval $-(\sqrt{2}-1)<x<\sqrt{2}-1$, where $|x|<\sqrt{2}-1<1 / 2$, switching for $\sqrt{2}-1<x<\sqrt{2}+1$ to $t=(1-x) /(1+x)$, which corresponds to $|t|<\sqrt{2}-1<1 / 2$. For greater values of $x$, one can use the $x \rightarrow 1 / x$ identities. For large negative values of $x$, i.e. $x<1-\sqrt{2}$, one can flip the sign of $x$ with Eq.(15) and then proceed as above.

In practice the transformation of Eq.(57) can lead to a large number of functions to be evaluated and hence it may be more profitable to apply this transformation only for values of $x$ that are much closer to one. If, on the other hand, nearly all H-functions of a given weight have to be evaluated for some value of $x$ one can use the turnover value of $\sqrt{2}-1$ in a rather profitable way.

The values in $x=1$ require some extra attention. These are actually needed rather frequently and hence there exists some literature on them. From Eq. (28) it should be clear that an H-function in $x=1$ can be expressed in terms of either $S$-sums or $Z$-sums in infinity. Hence much information can be found in [9], [5] and the papers they refer to. Ref. [6] gives a different method to evaluate these sums. Recently this method has been used by one of us (J.V.) to obtain all such sums up to weight 9 (see also footnote 2). For only nonnegative indices results have been obtained up to weight 11 [11]. When the first index of the $\mathrm{H}$-function (or the $S$-sum) is one, the value in $x=1$ (or the sum in infinity) will be divergent. Yet we have to consider these objects. As mentioned in the section on the algebra this can be done consistently only in terms of the sums. Hence the safest method is to rewrite the H-functions in $x=1$ immediately in terms of either $S$-sums or $Z$-sums. In the case that the weights are low enough, these can then be rewritten in terms of a limited set of 'fundamental constants'. 


\section{Mellin transforms}

At times one may need the Mellin transform of the Harmonic polylogarithms. In ref [6] a method is given to evaluate such transforms for a class of functions which is more or less the class of $\mathrm{H}$ functions. There is however one complication with Mellin transforms. Divergencies at $x=1$ must be extracted. This is because the Mellin transform is defined by

$$
\begin{aligned}
M(f(x), N) & =\int_{0}^{1} d x x^{N} f(x) \\
M\left(\frac{f(x)}{(1-x)_{+}}, N\right) & =\int_{0}^{1} d x \frac{x^{N} f(x)-f(1)}{1-x} \\
M\left(\frac{f(x) \ln ^{p}(1-x)}{(1-x)_{+}}, N\right) & =\int_{0}^{1} d x \frac{\left(x^{N} f(x)-f(1)\right) \ln ^{p}(1-x)}{1-x}
\end{aligned}
$$

in which the function $f$ is supposed to be finite for $x=1$ when the factor $1 /(1-x)_{+}$is present. Hence we have to pay attention to the powers of $\ln (1-x)$. They can be isolated with Eq. 23). After this extraction the remaining $\mathrm{H}$-functions are finite in $x=1$.

At this point we can attack the Mellin transforms. It is easy to obtain the lowest weight results:

$$
\begin{aligned}
\int_{0}^{1} d x x^{n} \mathrm{H}(0 ; x) & =-\frac{1}{(n+1)^{2}} \\
\int_{0}^{1} d x x^{n} \mathrm{H}(1 ; x) & =\frac{S_{1}(n+1)}{n+1} \\
\int_{0}^{1} d x x^{n} \mathrm{H}(-1 ; x) & =(-1)^{n} \frac{S_{-1}(n+1)}{n+1}+\frac{\ln (2)}{n+1}\left(1+(-1)^{n}\right)
\end{aligned}
$$

in which we have used that $\mathrm{H}(-1 ; 1)=-S_{-1}(\infty)=\ln (2)$. The higher weight results can be obtained by recursion. Like in ref [6] this is done by partial integration. We also exchange the sums immediately after each step so that we may do one of them immediately. The result is:

$$
\begin{aligned}
\int_{0}^{1} d x \sum_{i=n}^{\infty} \sigma^{i} x^{i} \mathrm{H}_{0, \vec{m}}(x) \frac{S_{\vec{p}}(i+1)}{(i+1)^{k}=} & \sigma \mathrm{H}_{0, \vec{m}}(1)\left(S_{\sigma(k+1), \vec{p}}(\infty)-S_{\sigma(k+1), \vec{p}}(n)\right) \\
& -\int_{0}^{1} d x \sum_{i=n}^{\infty} \sigma^{i} x^{i} \mathrm{H}_{\vec{m}}(x) \frac{S_{\vec{p}}(i+1)}{(i+1)^{k+1}} \\
\int_{0}^{1} d x \sum_{i=n}^{\infty} \sigma^{i} x^{i} \mathrm{H}_{1, \vec{m}}(x) \frac{S_{\vec{p}}(i+1)}{(i+1)^{k}}= & \sigma \mathrm{H}_{1, \vec{m}}(1)\left(S_{\sigma(k+1), \vec{p}}(\infty)-S_{\sigma(k+1), \vec{p}}(n)\right) \\
& -\int_{0}^{1} d x \sum_{i=n}^{\infty} x^{i} \mathrm{H}_{\vec{m}}(x)\left(\sigma S_{\sigma(k+1), \vec{p}}(i+1)\right. \\
& \left.-\sigma^{i} \frac{S_{\vec{p}}(i+1)}{(i+1)^{k+1}}-\sigma S_{\sigma(k+1), \vec{p}}(n)\right) \\
\int_{0}^{1} d x \sum_{i=n}^{\infty} \sigma^{i} x^{i} \mathrm{H}_{1, \vec{m}}(x) \frac{S_{\vec{p}}(i+1)}{(i+1)^{k}}= & \sigma \mathrm{H}_{1, \vec{m}}(1)\left(S_{\sigma(k+1), \vec{p}}(\infty)-S_{\sigma(k+1), \vec{p}}(n)\right)
\end{aligned}
$$




$$
\begin{aligned}
& -\int_{0}^{1} d x \sum_{i=n}^{\infty} x^{i} \mathrm{H}_{\vec{m}}(x)\left(\sigma(-1)^{i} S_{-\sigma(k+1), \vec{p}}(i+1)\right. \\
& \left.+\sigma^{i} \frac{S_{\vec{p}}(i+1)}{(i+1)^{k+1}}-\sigma(-1)^{i} S_{-\sigma(k+1), \vec{p}}(n)\right)
\end{aligned}
$$

The variable $\sigma$ is either 1 or -1 . This leaves only the evaluation of the H-functions in $x=1$. These values do not have to be finite. Only the $\mathrm{H}$-functions that are used in the subtraction in Eq.(73) are finite. This causes no problems provided the divergencies are regularized in the representation in terms of $S$-sums as explained before.

As an example we show here a nontrivial Mellin transform:

$$
\begin{aligned}
M\left(\frac{H_{1,-2,1,0}(x)}{1-x}, N\right)= & S_{1,-2,-1,2}(N)-2 S_{1,-2,-3}(N)+2 S_{1,5}(N) \\
& -\frac{1}{2} S_{1,-2}(N)\left(\zeta_{2} \ln (2)+\zeta_{3}\right)+S_{1,2}(N)\left(\frac{1}{2} \zeta_{2} \ln (2)-\zeta_{3}\right) \\
& +S_{1,1}(N)\left(4 \operatorname{Li}_{4}\left(\frac{1}{2}\right)+\frac{1}{6} \ln ^{4}(2)-\zeta_{2} \ln ^{2}(2)-\frac{13}{40} \zeta_{2}^{2}\right) \\
& +S_{1}(N)\left(\frac{9}{2} \zeta_{2} \zeta_{3}-\frac{83}{8} \zeta_{5}\right)-\frac{1}{24} \zeta_{2} \ln ^{4}(2)+\frac{7}{16} \zeta_{2} \zeta_{3} \ln (2) \\
& -\zeta_{2} \operatorname{Li}_{4}\left(\frac{1}{2}\right)+\frac{1}{4} \zeta_{2}^{2} \ln ^{2}(2)+\frac{447}{840} \zeta_{2}^{3}-\frac{157}{32} \zeta_{3}^{2}+\frac{7}{2} S_{-5,-1}(\infty)
\end{aligned}
$$

The sum in the last term is irreducible.

In the case that the weight of the terms too large (currently larger than 9) it becomes rather hard to obtain the values for the H-functions in $x=1$ or alternatively for the $S$-sums in infinity. Because the algebra for the H-functions in $x=1$ is different from the algebra for the $\mathrm{H}$-functions for general values of $x$ there may be large numbers of $\mathrm{H}$-functions left that each are divergent at $x=1$. The reason is that some algebraic work is done first with the general algebraic rules and has to be 'undone' with the rules for $x=1$. The relations that make the divergences cancel may not be easy to find. One can still obtain numerical results however.

If one is faced with higher weights one may proceed as follows. The H-functions in $x=1$ are first expressed in terms of $S$-sums in infinity. Then the shuffle algebra for the $S$-sums is used to extract the divergencies in a way that is similar to how this is done for the powers of $\ln (1-x)$ for the H-functions. Because the divergences have to cancel each other, all divergent terms should disappear, even though we may not have the algebraic methods to prove this for the case at hand. The remaining finite expression can in principle be evaluated numerically.

Inverse Mellin transforms are now relatively easy. As pointed out in ref [6] each $S$-sum has a single most complicated original function in terms of $\mathrm{H}$-functions in which we can define 'most complicated' by function with largest weight or in the case of identical weights the largest number of nonzero indices. And actually one can obtain the relation between the $S$-sum of which one needs the inverse Mellin transform and this most complicated H-function from the recursion relations in 
Eq.(75). Hence the algorithm is clear:

- Locate the most complicated $S$-sum(s).

- Construct the corresponding H-function(s) in $x$-space.

- Add it and subtract it.

- Make the Mellin transform of the subtracted version. This will cancel the original $S$-sum.

- Repeat the above steps until there are no more $S$-sums remaining.

- Multiply the remaining constant terms by $\delta(1-x)$.

This algorithm will properly terminate. It has only one problem: Some Mellin transforms have a factor $(-1)^{N}$ and some don't. What if we take an $S$-sum which should have a factor $(-1)^{N}$ but we omit it? Here we have to realize that the inverse Mellin transform is to be constructed from either all even or from all odd moments only. Hence we have to specify whether $N$ is even or odd. This will give a value to $(-1)^{N}$. Hence the only thing that remains is to give the relation between an $S$-sum and the most complicated H-function that contributes to it.

- If the number of negative indices is odd, there will be a factor $1 /(1+x)$, otherwise there will be a factor $1 /(1-x)_{+}$.

- Next copy the index field to the H-function.

- Working from the rightmost index to the left, each index will get a sign that is the combination of its old sign and the signs of all indices to the left of it.

- There will be an additional overall sign on the term which is the sign of the last index.

- There will be an additional overall sign on the term which is $(-1)^{w-1-d}$ in which $w$ is the weight of the $S$-sum and $d$ its depth (which is the number of nonzero indices).

- Each negative index in the current configuration will give a minus sign to the term.

We will give two examples of weight 7 functions. First and example that involves subtractions with $\ln ^{2}(1-x)$ in the Mellin transform:

$$
\begin{array}{r}
S_{1,1,2,1,2}(N) \rightarrow \frac{1}{1-x}\left(-\mathrm{H}_{1,1,2,1,0}(x)-\frac{1}{2} \mathrm{H}_{1,1}(x) \zeta_{2}^{2}\right) \\
+\delta(1-x)\left(-\frac{3}{2} \zeta_{2} \zeta_{5}-\frac{7}{5} \zeta_{2}^{2} \zeta_{3}+17 \zeta_{7}\right)
\end{array}
$$


In this case there is no difference for even values of $N$ and for odd values of $N$. However the next example is different. For even values of $N$ we have

$$
\begin{aligned}
S_{-1,1,-2,1,2}(N) \rightarrow \frac{1}{1-} & \left(-\mathrm{H}_{-1,-1,2,1,0}(x)+2 \mathrm{H}_{-1,-1,0,0,0,0}(x)-2 \mathrm{H}_{0,0,0,0,0,0}(x)\right. \\
& +\frac{1}{2} \mathrm{H}_{-1,-1}(x) \zeta_{2}^{2}-\mathrm{H}_{0,0}(x) \zeta_{2}^{2} \\
& +\mathrm{H}_{-1}(x)\left(\frac{1}{16} \zeta_{2} \zeta_{3}+\zeta_{2}^{2} \ln (2)+\frac{67}{64} \zeta_{5}\right) \\
& +\mathrm{H}_{0}(x)\left(-\frac{1}{8} \zeta_{2} \zeta_{3}-2 \zeta_{2}^{2} \ln (2)-\frac{67}{32} \zeta_{5}\right) \\
& \left.-\frac{1}{8} \zeta_{2} \zeta_{3} \ln (2)-\frac{3}{2} \zeta_{2}^{2} \ln ^{2}(2)-\frac{307}{560} \zeta_{2}^{3}+\frac{157}{128} \zeta_{3}^{2}+\frac{21}{64} \zeta_{5} \ln (2)-\frac{5}{4} \sigma_{6}\right) \\
+ & \frac{1}{1+x}\left(\left(\mathrm{H}_{1}(x)+2 \mathrm{H}_{0}(x)\right)\left(\frac{1}{16} \zeta_{2} \zeta_{3}-\frac{53}{64} \zeta_{5}\right)\right. \\
& \left.-\frac{61}{560} \zeta_{2}^{3}+\frac{35}{128} \zeta_{3}^{2}+\frac{93}{64} \zeta_{5} \ln (2)-\frac{3}{4} \sigma_{6}\right) \\
+ & \delta(1-x)\left(-\frac{1}{16} \zeta_{2} \zeta_{3} \ln ^{2}(2)-\frac{957}{224} \zeta_{2} \zeta_{5}+\frac{1}{120} \zeta_{2} \ln ^{5}(2)-\zeta_{2} \operatorname{Li}_{5}\left(\frac{1}{2}\right)\right. \\
& -\frac{93}{140} \zeta_{2}^{2} \zeta_{3}-\frac{1}{12} \zeta_{2}^{2} \ln ^{3}(2)-\frac{29}{280} \zeta_{2}^{3} \ln (2)-\frac{1355}{896} \zeta_{3}^{2} \ln (2) \\
& \left.-\frac{197}{64} \zeta_{5} \ln ^{2}(2)+\frac{37215}{3584} \zeta_{7}+\frac{19}{28} \ln (2) \sigma_{6}-\frac{10}{7} \sigma_{7, a}+\frac{29}{14} \sigma_{7, b}\right)
\end{aligned}
$$

in which

$$
\begin{aligned}
\sigma_{6} & =S_{-5,-1}(\infty) \\
\sigma_{7, a} & =S_{-5,1,1}(\infty) \\
\sigma_{7, b} & =S_{5,-1,-1}(\infty)
\end{aligned}
$$

In the case of odd $N$ the terms with $1 /(1+x)$ change sign. As one can see these formulae can become rather involved, even though the number of terms is rather small compared to the number of functions that exist in $x$-space for this weight.

In the case that sums of a higher weight are considered one may not be able to substitute the values of the $\mathrm{H}$-functions at $x=1$. The same considerations as for the Mellin transforms can be used to obtain an answer that can at least be evaluated numerically. In general the formulae will of course be much lengthier.

Acknowledgements. One of the authors (E.R.) wants to thank the Alexander von Humboldt 
Stiftung for the generous support of his stay at Karlsruhe. The other author (J.V.) would like to thank the Programa "Catedra" of the Fundacion BBV for support during the part of this work which was done at the Universidad Autónoma of Madrid.

We like to thank S. Moch for discussions.

\section{References}

[1] G. Racah, Sopra l'irradiazione nell'urto di particelle veloci, Nuovo Cimento 11,461 (1934).

[2] K.S. Kölbig, J.A. Mignaco and E. Remiddi, On Nielsen's generalized polylogarithms and their numerical calculation, B.I.T. 10, 38 (1970); see also the Appendices of the papers by R. Barbieri, J. A. Mignaco and E. Remiddi, Nuovo Cimento 11A, 824 and 865 (1972).

[3] N. Nielsen, Der Eulersche Dilogarithmus und seine Verallgemeinerungen, Nova Acta Leopoldina (Halle) 90 (1909) 123.

[4] L. Lewin Dilogarithms and Associated Functions, North Holland 1958; Polylogarithms and Associated Functions, North Holland 1981.

[5] J. M. Borwein, D. M. Bradley, D. J. Broadhurst and P. Lisonek: 'Special values of Multidimensional Polylogarithms', CECM Research Report 98-106.

[6] J.A.M. Vermaseren, hep-ph/9806280.

[7] J.A.M. Vermaseren, Symbolic Manipulation with FORM, version 2, Computer Algebra Nederland, Amsterdam (1991). Version 3, which is used for the programs in this paper, will be released shortly.

[8] D. J. Broadhurst, D. Kreimer Phys. Lett. B393 (1997) 403.

[9] J.M. Borwein, D.M. Bradley, D.J. Broadhurst, hep-th/9611004, The Electronic Journal of Combinatorics, Vol. 4, No. 2 (Wilf Festschrift), 1997, \#R5.

[10] E. B. Zijlstra and W. L. van Neerven, Nucl. Phys. B383 (1992) 525; E. B. Zijlstra, PhD thesis, RX1449, Leiden (1993).

[11] D. J. Broadhurst, private communication. 